\title{
Carnets
}

Revue électronique d'études françaises de l'APEF

Première Série - 1 Numéro Spécial | 2009

Cultures littéraires : nouvelles performances \& développement

\section{"The storyteller is deep inside every one of us..." Récit et communication: des performances durables}

Maria de Jesus Cabral

\section{(2) OpenEdition}

Journals

Édition électronique

URL : http://journals.openedition.org/carnets/3475

DOI : $10.4000 /$ carnets.3475

ISSN : 1646-7698

Éditeur

APEF

Édition imprimée

Date de publication : 1 juin 2009

Pagination : 53-63

Référence électronique

Maria de Jesus Cabral, «"The storyteller is deep inside every one of us..." Récit et communication: des

performances durables », Carnets [En ligne], Première Série - 1 Numéro Spécial | 2009, mis en ligne le

16 juin 2018, consulté le 04 mai 2019. URL : http://journals.openedition.org/carnets/3475 ; DOI :

$10.4000 /$ carnets. 3475

Carnets est mis à disposition selon les term
d'utilisation commerciale 4.0 International. 


\title{
"THE STORYTELLER IS DEEP INSIDE EVERY ONE OF US...” Récit et communication: des performances durables
}

\author{
MARIA DE JESUS CABRAL \\ FCT/FLUC \\ mariajesu@gmail.com
}

\begin{abstract}
Résumé
Nous vivons en un temps curieux : d'un côté, un éclatement des systèmes d'information globalisés, créant de nouveaux supports et de nouveaux modes de communication, de nouveaux concepts et de nouveaux rapports au temps, à l'espace, à autrui... De l'autre, un déficit sans précédent du discours et une instrumentalisation grandissante de ressources séculaires d'interaction humaine, comme le récit et la narration qui lui est corrélée. En nous inspirant de la fin du discours de Doris Lessing à l'occasion de la remise du prix Nobel de la littérature en 2007, nous nous proposons d'abord d'interroger la notion de storytelling à la lumière de la notion de fiction, telle qu'elle a été suggérée par Mallarmé ; dans un second temps, nous présenterons quelques usages et mésusages actuels du storytelling, à partir de l'ouvrage homonyme de Christian Salmon (2007), tout en essayant d'en déchiffrer quelques risques et enjeux; pour finir, nous interrogerons les potentialités performatives offertes par une pratique en devenir dans plusieurs domaines du savoir. À quel point pourrait-elle être "notre Phénix"?
\end{abstract}

\section{Abstract}

We live in a curious time: on one side the unprecedented rupture of global information systems, creating new forms and new modes of communication, new concepts and new relationships of time, space and of the other on, on the other side the difficulty to communicate, and an increasing instrumentalization of human secular resources such as stories and its connected term narrative. We will attempt to answer these questions with the inspirational words of Doris Lessing's Nobel lecture (2007). Firstly, we will question them according to the notions of fiction, as was first suggested by Mallarmé. Secondly, we will present different current uses and false uses of "storytelling" its risks and stakes, based on the homonym work of Christian Salmon (2007). And lastly, we will try to clarify the potential performatives covered by this secular practice. To what extent is this "our Phoenix"?

Mots-clés: Récit, Storytelling, Communication, Fiction, Performance

Keywords: Story, Storytelling, Communication, Fiction, Performance

Maria de Jesus Cabral, "'The storyteller is deep inside every one of us...' Récit et communication: des performances durables", Carnets, Cultures littéraires: nouvelles performances et développement, ㄲo spécial, automne / hiver 2009, pp. 53-63. 


\begin{abstract}
The storyteller is deep inside everyone of us. The storymaker is always with us. Let us suppose our world is attacked by war, by the horrors that we all of us easily imagine. Let us suppose floods wash through our cities, the seas rise ... but the storyteller will be there, for it is our imaginations which shape us, keep us, create us for good and for ill. It is our stories, the storyteller that will recreate us, when we are torn, hurt, even destroyed. It is the storyteller, the dream-maker, the myth-maker, that is our phoenix, what we are at our best, when we are our most creative.
\end{abstract}

Doris Lessing ${ }^{1}$

Dans une œuvre mémorable de la pensée littéraire et philosophique moderne, JeanPaul Sartre a écrit que l'homme est "condamné à être libre". À la fin de son discours de réception du Nobel de la Littérature en 2007, cité ci-dessus, l'écrivaine Doris Lessing suggère que l'homme est 'condamné' à l'histoire. En deçà de l'opposition admise par la graphie entre history et story ${ }^{2}$, l'auteure semble jouer sur l'ambiguïté sémantique du mot, évoquant, dans le même contexte, aussi bien l'histoire qui s'écrit avec un petit h comme un grand " $\mathrm{H}$ " - avec une grande hache, pourrait-on le dire également, tant il est vrai que la littérature a depuis toujours partie liée au sort de l'humanité, aussi tranchantes que se soient révélées ses "horrors", devant nos yeux ou "deep inside of us ". Le propos de Doris Lessing laisse bien entendre pourtant que si l'œuvre emprunte au réel, elle vit de et dans un entredeux mobile, dans un libre à venir, dans un "quelque part en dehors du monde ", d'où le rapport synonymique "storyteller" / "dream-maker" / "myth-maker". Dans ce sens aussi, le lecteur est à son tour un "story-maker", qui transforme ce matériau, tel un alchimiste, en y incorporant ses émotions et son imagination créative, créatrice. Tout le discours de Doris Lessing est marqué par le cogito énoncé ci-dessus "for it is our imaginations which shape us, keep us, create us".

C'est par la magie des mots que le lecteur devient à son tour " fabricant ". À l'instar de celui du poète - au sens étymologique du mot - le travail du lecteur se déroule dans un univers autre que celui du monde connu, projeté par le jeu de l'imagination, "reine des facultés comme l'a écrit Baudelaire dans son "Salon de 1859 " (Baudelaire, 1968 : 396), "sortilège " consubstantiel à la poésie, comme le rappelle Mallarmé dans le poème critique "Magie" (Mallarmé, 2003: 250) et qu'il a rehaussé dans la performance minimale de la danseuse Loïe Fuller, dans les poèmes critiques qu'il lui a dédiés. En effet, à la façon "d'un

\footnotetext{
1 Discours de Doris Lessing lu par son éditeur Nicholas Pearson, le 7 décembre 2007. Voir http://nobelprize.org/nobel_prizes/literature/laureates/2007/lessing-lecture_en.html. Consulté le 04 janvier 2008.

${ }^{2}$ La lexicologie de la langue anglaise permet de distinguer 'history' et 'story', selon l'acception véridique ou fictionnelle visée.
} 
haut et vivant signe" (Mallarmé, 2003 : 275), la danseuse américaine déploie au travers ses jeux de voile et de lumière des interstices de sens, source de toutes les virtualités, où la réalité s'estompe laissant "la scène libre, au gré de la fiction" (Mallarmé, 2003 : 313). Autour de la danseuse disparaît "la traditionnelle plantation de stables ou opaques décors" (idem) et "l'art jaillit incidemment, souverain" (idem) dans ce que le poète appelle le décor "latent" "trésor des imaginations" (Mallarmé, 2003: 175). Et c'est ainsi que le Ballet devient "la forme théâtrale de poésie par excellence" (idem). Si Mallarmé, dans sa conception d'un théâtre poétique, réaffirme souvent la "scène de l'esprit" ou d'un "théâtre intérieur " comme lieu par excellence d'élaboration du sens, c'est qu'il considère que " rien " n'est donné dans la poésie, le sens est toujours un lieu qui reste à découvrir - au double sens du terme. Lire, c'est participer à cette découverte poétique du sens, ce qui sollicite hautement le jeu libre de l'imagination et de la fiction, comme le suggère cette image qui ouvre le poème "Eventail de Madame Mallarmé" :

\author{
Avec comme pour langage \\ Rien qu'un battement aux cieux \\ Le futur vers se dégage \\ Du Logis très précieux... (Mallarmé, 1998: 30)
}

Il est clair que pour Mallarmé la "Fiction ou Poésie", comme il l'a suggéré dans "Solennité" (Mallarmé, 2003 : 203) relève du langage et du principe mystérieux de l'esprit humain, comme il ressort dès les ébauches de ses " notes" sur langage ${ }^{3}$ :

Le langage lui est apparu l'instrument de la fiction : il suivra la méthode du langage. Le langage se réfléchissant. Enfin, la fiction lui semble être le procédé même de l'esprit humain - c'est elle qui met en jeu toute méthode, et l'homme est réduit à la volonté (Mallarmé, 1998: 504).

Cette recréation ou opération alchimique que Doris Lessing évoque dans son discours - "notre patrimoine d'histoires est né dans le feu, la magie, le monde des esprits" rappelle-t-elle - est sous-tendue par la symbolique du phénix et tout ce qu'elle convoque comme pulsion organique, comme devenir perpétuel, de l'homme, du divin, de la création. L'imagination se fait fort de reconstruire, de recréer le monde. Mais le moyen dont le poète se sert pour que cette métamorphose soit possible c'est le langage : le langage des signes, mais aussi, par le jeu du sens, le langage des relations, le langage du silence, le langage de

\footnotetext{
${ }^{3}$ Destinées à l'origine à une thèse de linguistique. La première référence se trouve dans une lettre de décembre 1869 où Mallarmé confie "retrouvant en face d'un livre [le Discours de la Méthode] toute ma pensée, je m'étais initié à des études (de linguistique), mon refuge, le cas échéant ". II fait encore référence à ce travail, un peu plus loin, comme des études de "Doctorat" (Mallarmé, 1995: 457).
} 
la transcendance, capable de prendre le relais du monde, de reformuler la liaison des mots aux choses sous un angle nouveau, métaphorique, insolite, subversif, contraignant...

\section{“Não sei, ama, onde era, / Nunca o saberei...” (Fernando Pessoa, “ Conta-me contos, ama")}

Le phénix est à la fois l'emblème de l'histoire, du récit et de la narration ${ }^{4}$ qui s'associent pour faire renaître à l'infini la vitalité créatrice de la production artistique. Mais le storytelling ou "art de raconter" implicite dans cette désignation et qui reste une des plus anciennes pratiques de l'humanité, est un acte de parole combinant savoir et dialogue, dans un espace-temps précis. En ce sens, l'acte de narrer est un mouvement messager entre émetteur et récepteur en un processus de (re)création irréversible et éphémère, qui se passe dans le hic et nunc de la communication. Qu'il se déroule dans un environnement familial ou plus largement ouvert, dans la proximité de son interlocuteur ou en groupe, le succès dépendra de la performance contextuelle du conteur : la façon dont il verbalise, dont il place les sonorités et le rythme, la gestuelle qui accompagne la transmission du récit. Comme l'a bien observé Brunhilde Biebuyck, raconter implique "la construction de toute une ambiance relationnelle /.../ le récit en lui-même ne représente qu'un élément" (Biebuyck, 1991 : 110). En outre, dans sa performance devant le public, il est tout un travail d'ajustement et d'adaptation au contexte précis de la communication. Rappelant le concept iserien de "structures d'appel " du texte, nous pouvons dire que le conteur doit les faire correspondre aux intensités et aux modalisations de son discours et de ses gestes mêmes, les signes se transmuant en sensations, dans une sorte de théâtralité où dire et montrer ne doivent faire qu'un.

En nous inspirant à la fois de Genette et de Bachelard ${ }^{5}$ nous dirons que l'acte de raconter est mouvement dialectique entre l'immanence de l'œuvre et sa transcendance, entre "l'objet littéraire" et le mouvement du sens qui procède celui-ci par le cours de la parole ${ }^{6}$. Le 'storyteller' se constitue donc en conducteur et agent d'un patrimoine littéraire et culturel - parfois (très distant) de notre ici/maintenant, si l'on songe aux récits mythiques classiques ou médiévaux - inscrit dans l'histoire et dans l'imaginaire qui l'a façonné, qui renaît dans la communication poétique. C'est pour cette capacité à "demeurer le même et

\footnotetext{
${ }^{4}$ Étant donnée l'hétérogénéité terminologique des désignations 'histoire', 'récit', 'narration' nous suivrons le sens narratologique respectif défini par Gérard Genette dans "Discours du récit" (Figures III) : " histoire /est/ la signification ou contenu narratif /.../ récit proprement dit le signifiant, énoncé, discours ou texte narratif lui-même, et narration l'acte narratif producteur" (Genette, 1972: 72).

${ }^{5}$ Et, respectivement de leurs ouvrages L'œuvre de l'art. Immanence et transcendance (1994) et Poétique de la rêverie (1971).

6 Dans l'œuvre citée précédemment, Bachelard postule que "la poésie est un des destins de la parole" (Bachelard, $1971: 3$ ).
} 
devenir toujours nouveau", comme l'a si bien dit Umberto $\mathrm{Eco}^{7}$, que le storytelling nous reflète "au mieux de nous-mêmes et de notre créativité", pour revenir au discours de Doris Lessing. Parce qu'il est le produit d'un mystère, d'une façon de dire ce qui ne peut être appréhendé autrement que dans le mystère, le récit est toujours ouvert, toujours déchiffrable à nouveau. C'est la magie du phénix, le secret " inclus et latent" prêt à se manifester quels que soient les temps où les espaces, dans toute sa force, dans toute sa fiction - au double sens étymologique - fabrication et feintise. Un masque et une révélation à la fois, comme le suggère ce fragment des "Mélanges" de Paul Valéry, intitulé "Magie" :

Fermez les yeux, dit l'homme. Bien. Imaginez Irma. Fortement, nettement. Vous la voyez ? Bien. À présent, ouvrez-les !

Irma était devant lui.

(Rideau) (Valéry, 1957 : 309)

\section{“Enfants ! faites attention aux baobabs!” (Le Petit Prince, Saint-Exupéry)}

De nos jours l'art de narrer n'a pas tari sa veine : il est partie intégrante de notre horizon culturel et éducationnel (au sens large), de la plus tendre enfance à toutes sortes de manifestations plus ou moins transversales autour du livre et de la lecture.

Or "tout ce qui brille n'est pas de l'or". Cette apologie du récit et ses potentialités esthétiques et éthiques - tant du point de vue de sa production comme de sa réception - va de pair avec un usage ou mésusage actuel qui ramène le storytelling à une sorte d'instrumentalisation de son objet comme de ses fins. Le mot storytelling illustre en effet le cas somme toute courant d'un mot anglais repris à son contexte d'origine (récit), mais utilisé avec une acception toute particulière. II ne s'agit pas du récit au sens narratologique défini cidessus, mais plutôt story au sens de petites histoires ou anecdotes utilisées comme stratégie de persuasion, de contrôle et de pouvoir. Le phénomène est dès lors favorisé soit par la prédisposition humaine à l'histoire, soit par le principe structurel du récit, qui offre un moyen des plus efficaces pour organiser et stocker de façon systématique l'information. II tire par ailleurs parti d'un autre facteur relevant dans l'acte communicationnel qui est l'émotion, véritable "ferment" de l'acte communicationnel et qui est la base et la force motrice du comportement. Les travaux récents dans le domaine de la neuroscience ont bien révélé le rôle des émotions et du sentiment dans la perception du monde et dans la prise de décisions humaines ${ }^{8}$, somme toute dans nos activités les plus diverses.

\footnotetext{
${ }^{7}$ Dans son article "Le problème de la réception" in Eco, $1970: 14$.

${ }^{8}$ Nous renvoyons aux travaux pionniers d'António Damásio L'erreur de Descartes (1995) ; Le sentiment même de soi (1999), Paris : Odile Jacob.
} 
L'emploi 'élémentaire' du storytelling ou sa face 'universel reportage', expressions utilisées par Mallarmé à la fin du XIX ${ }^{\text {ème }}$ siècle pour caractériser une littérature en pleine crise de son objet, le langage, à l'avènement de la culture massifiée ${ }^{9}$, a atteint de nouveaux développements et des performances variables dans la galaxie postmoderne fortement médiatisée, que l'on sait marquée, entre autres aspects de sa condition, selon le mot de J.-F. Lyotard $^{10}$, par l'efficacité empirique. À l'heure de la mondialisation, la culture aurait paradoxalement perdu sa dimension humaniste et cosmopolite, centrifuge, pourrions-nous dire, pour devenir instrumentale, fonctionnaliste, et, dans le même ordre d'idées, centripète. Tel que l'a observé le même auteur, "le rapport au savoir n'est pas celui de la réalisation ou de la vie de l'esprit ou de l'émancipation de l'humanité ; c'est celui des utilisateurs d'un outillage conceptuel et matériel complexe, et des bénéficiaires de la performance" (Lyotard, $1979: 86)$.

Le storytelling au sens des nouveaux usages ou mésusages du récit s'insère dans ces nouveaux paramètres de la postmodernité. C'est ce que vient illustrer l'ouvrage homonyme de Christian Salmon, publié en 2007, au sous-titre suggestif La machine à fabriquer des histoires et à formater les esprits. D'où vient cette pratique, à la lisière d'un 'mauvais genre' ? Le livre de C. Salmon, situe ses origines aux États-Unis au milieu des années 90. A cette période, "le tournant narratif des sciences sociales coïncide avec l'explosion d'Internet et les avancées des nouvelles techniques d'information et de communication", explique l'auteur. "C'est l'avènement de la consommation comme seul rapport au monde", ajoute-t-il, énonçant ainsi deux caractéristiques majeures de la culture postmoderne: la consommation et le rapport aux nouvelles technologies. Un éventail éclectique de businessmen - qu'ils soient publicitaires, politiques, financiers - découvrent l'efficacité marchande du storytelling et exploitent la "story" comme une nouvelle monnaie du management, servant à manipuler les "consommateurs ", rendant floue la limite entre le réel et la fiction, entre la vérité et le mensonge. Ces nouveaux 'conteurs' tirent parti des structurations mentales, des attentes vis-à-vis l'imaginaire collectif pour "oriente[r] les flux d'émotions" (Salmon, 2007 :16). En faisant référence à Roland Barthes et aux "parcours de reconnaissance" que ce dernier "décryptait dans l'activité narrative" (idem: 17), l'auteur montre que le storytelling se fonde sur des "engrenages narratifs, suivant lesquels les individus sont conduits à s'identifier à des modèles et à se conformer à des protocoles" (idem). La force du modèle tient sans doute à son étonnante transversalité. C. Salmon

\footnotetext{
${ }^{9}$ Les réflexions énoncées dans "Crise de Vers" (1891) sont à cet égard révélatrices, et anticipent celles de noms

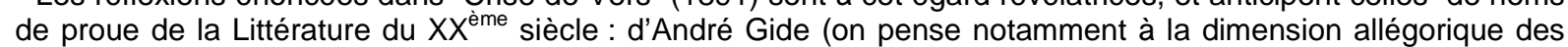
Faux-Monnayeurs présente aussi bien dans le roman éponyme de 1925 que dans le Journal qui l'a précédé) à Paul Valéry (les réflexions toujours d'actualité de ses Cahiers), aux apories sur le langage et la représentation énoncées par les Nouveaux Romanciers des années 1950 dont les échos se répercutent par exemple dans L'usage de la parole (1980) de Nathalie Sarraute, jusqu'à nos jours.

$10 \mathrm{~J}$ 'ai notamment en vue son livre La condition postmoderne de 1979.
} 
illustre le phénomène en puisant des exemples aussi bien dans le domaine de la nouvelle "économie fiction" (2007: 75) que dans les "entreprises récitantes" (Salmon, 2007: 101). Qu'elles se nomment Renault, McDonald's, Disney ou Nike, le récit y tient lieu de stratégie commerciale qui "structure et formate la communication, à l'intention des consommateurs comme des actionnaires" (idem: 102).

Le storytelling se rapproche donc des origines orales du conte, mais cette 'prise de parole' est un outil idéologiquement orienté : “il ne s'agit pas seulement de 'raconter des histoires' cacher la réalité d'un voile de fictions trompeuses, mais aussi de faire partager un ensemble de croyances à même de susciter l'adhésion et d'orienter le flux des émotions " (idem). Dès lors, on comprend que cette pratique du monde des affaires soit devenue l'instrument privilégié du domaine politique, dont C. Salmon décrypte, avec beaucoup d'humour, les " mises en histoires " (Salmon, 2007 : 111) du "grand narrateur Reagan " aux "disciples Clinton et Sarkozy" (idem: 126). A l'arrière-fond de la "guerre virtuelle" ou "storytelling à Bagdad" (idem: 141), l'auteur montre les avatars de la collaboration Hollywood-Pentagone sous l'égide de l'Institute for Creative Technologies (ICT) basé sur les " technologies de simulation mises au point par les industries du jeu vidéo et les experts en storytelling d'Hollywood, afin de repenser un nouveau système d'entraînement /.../ militaire " (idem: 143). De là aux “armes de distraction massive" (Salmon, $2007: 157)$ il n'y avait qu'un pas. Christian Salmon suit le fil de ce qu'il appelle une "realpolitik de la fiction" (Salmon, 2007 :172) en prenant l'exemple performant d'un "story spinner" comme Karl Rove, conseiller du président Georges W. Bush qui serait le "fabricant" de la fiction des "armes de destruction massive" qui ont entraîné le monde dans la guerre contre l'Irak. Ses propos témoignent bien de la confusion délibérée entre la réalité et la fiction : "nous sommes les acteurs de l'histoire", " nous sommes un empire /.../ nous créons notre propre réalité " (idem) sont autant d'expressions qui, au jugement de C. Salmon, "semblent s'élever d'une scène de théâtre plutôt que d'un bureau de la Maison-Blanche" (idem), contrariant aindi l'idée selon laquelle les décisions politiques reposeraient sur des critères objectifs et rationnels. L'auteur identifie ce procédé auquel les citoyens américains se sont vus confrontés comme " un nouvel ordre narratif" - "NON" - qui explique notamment que certaines chaînes de télévision comme la Fox News aient substitué des "stories /.../ aux faits réels" (Salmon 2007 : 179), et soient devenues une sorte de structure souterraine du pouvoir politique, particulièrement efficace aux Etats-Unis au cours des années "Double U", mais ayant progressivement gagné l'Europe. La campagne pour l'élection présidentielle française de 2007 appelle à son tour les stratégies du storytelling made in USA. Les déclarations de Henri Guiano au Monde sur sa contribution à la campagne présidentielle de Nicolas Sarkozy en accusent la tendance: "la politique c'est une histoire partagée par ceux qui la font et ceux à qui elle est destinée. On ne transforme pas un pays sans être capable d'écrire et de raconter 
une histoire" (2007 : 200) rappelle C. Salmon, détectant chez les principaux candidats le même souci d'adhésion émotionnelle au détriment de l'argumentation rationnelle. Cette disposition habite nombre de prestations électorales, favorisant l'émergence d'une "ère nouvelle de la démocratie, que l'on pourrait qualifier de 'postpolitique'" conclut C. Salmon (2007 : 210). L'acte de raconter devient alors une "arme de distraction massive " ou 'soft power' - quelle actualisation du " panem et circenses " de la Rome césarienne !

Mais ce que l'on peut observer dans ce storytelling que Christian Salmon accule au mondialisme nord-américain c'est qu'il révèle une situation proprement troublante dans la mesure où il repose sur la "banalisation ", voire la " dénaturation" de la matrice comme du principe réceptif et actif du récit, puisque le récepteur n'est pas appelé à la co-création du sens mais à l'inertie, au consentement et à la passivité. La dimension esthétique se perd dans le bouillon superficiel de "I'universel reportage ", du bavardage et du vide sidéral du discours. Le livre Storytelling...., il semble utile de le rappeler, s'inscrit dans le prolongement, notamment de Verbicide, du bon usage des cerveaux humains disponibles où l'auteur réfléchit sur les enjeux de la profusion de l'information comme facteur décourageant la compréhension et l'interprétation ${ }^{11}$. Christian Salmon y emploie le terme verbicide pour définir la situation de crise qui s'est installée dans le langage, à l'ère de la globalisation : "Le langage s'est démonétisé. II a cessé d'avoir une vie stable et reconnue /.../ il n'a plus cours qu'au marché noir des medias, où abonde la fausse monnaie des rumeurs et des anecdotes" (Salmon, 2005 : 11).

Les trois "s" du storytelling - simulation, séduction et stimulation - sont autant d'armes d'une nouvelle doxa bien enracinée - quel baobab - dans le monde marchand, global, largement mass-médiatisé où "I'homme des temps modernes " est pris non plus ou pas seulement dans l'engrenage des machines, comme le montra Chaplin, mais dans celui, tout aussi dynamique et peut-être issu du précédent : celui de la consommation fat and fast et de l'hédonisme narcissiste.

La relation avec la partie précédente est évidemment - et nous devons le souligner essentielle. Plus le récepteur sera doté d'un répertoire culturel, littéraire et langagier, plus il sera muni d'une compétence interprétative - celle-là même qui s'acquiert et se développe notamment au contact avec les formes artistiques - mieux il sera à-même de 'lire' et de décrypter de façon plus critique les sens essaimés et de 'déconstruire' les intentions manipulatrices.

\footnotetext{
${ }^{11}$ Partant de Walter Benjamin, Salmon constate qu'aucun événement "n'arrive plus jusqu'à nous sans être accompagné d'explications/.../ presque tout sert à l'information." (Verbicide..., p. 9).
} 


\section{“Allier, ne confondre” (Stéphane Mallarmé)}

L'appropriation du récit n'est pas une tendance spécifiquement postmoderne. Depuis Homère aux chansons de geste, des fables et mythes païens ou religieux, des contes et légendes de la tradition orale aux adaptations modernes, l'utilisation de l'histoire pour exemplifier, pour démontrer, pour influencer les comportements individuels ou collectifs qu'ils soient d'ordre religieux, ethnique, idéologique ou simplement pédagogique - est un phénomène intemporel. Dans un article publié dans la revue Scientific American en octobre 2007, et intitulé "The really hard science" "12, Michael Shermer, connu dans la sphère médiatique pour ses positions sceptiques, envisage le lien entre les sciences dites "dures" et les sciences humaines, sous fond de ses recherches sur Darwin. La position du chroniqueur est explicite dans le sous-titre de son article "To be of true service to humanity, science must be an exquisite blend of data, theory and narrative" et illustrée d'une perspective biologique, évolutionniste:

As primates, humans seek patterns and establish concepts to understand the world around us, and then we describe it. We are storytellers. If you cannot tell a good story about your data and theory - that is, if you cannot explain your observations, what view they are for or against and what service your efforts provide - then your science is incomplete (Shermer, 2007).

Dépassant le clivage fait / fiction et le postulat de l'objectivité scientifique, M. Shermer prône que le récit joue un rôle décisif dans le contexte de la communication scientifique. Sa réflexion sur cette question présente des arguments qui viennent contrebalancer le problème du mésusage du récit, que nous avons abordé précédemment. C'est là une autre facette du storytelling et ses potentialités, à la fois comme médiation, rendant l'exposé plus clair, plus cohérent, plus captivant - plus efficace, en un mot - et comme structure logique de cheminement, qui doit prévaloir pour rendre le message intelligible. Plus grande est la complexité des données ou des théories présentées, plus elle doit susciter les capacités d'organisation et d'intelligibilité du communicant, qu'il soit conférencier, orateur ou enseignant. Dans ce sens, la communication en contexte pédagogique peut tirer des potentialités de progression séquentielle du récit, utiliser la narration pour impliquer son récepteur, et l'histoire, voire la petite histoire pour détendre l'atmosphère, retenir l'attention, gérer les transitions.... Même en contexte universitaire, l'enseignement ne saurait se soustraire d'un rôle qui est à la fois d'accompagnement et de motivation. L'étymologie du mot pédagogie nous l'indique : conduire, mener, accompagner. La mission n'est pas aisée,

\footnotetext{
${ }^{12}$ Disponible à l'adresse http://www.scientificamerican.com/article.cfm?id=the-really-hard-science.
} 
puisqu'il faut allier savoir et savoir-faire, connaissance et capacité de transmission. Sans rendre inintelligible, sans tomber dans la vulgarisation. Dans le même ordre apologique, la littérature, dans toutes ses formes et dans tous ses états est un excellent moyen de développement des capacités cognitives et du raisonnement analogique, soit une dimension fondamentale à tous les secteurs de l'activité humaine.

II est en effet bien des façons d'appréhender le monde : par des intuitions personnelles, ou des révélations mystiques, par diverses formes de croyances religieuses, par la voie de la science par l'art et la littérature. Parmi ces différentes approches, la Littérature est peut-être celle qui peut offrir le champ d'action le plus vaste car elle est fondée sur tous les préceptes antérieurs : une expérience, susceptible d'être communiquée, partagée ; elle peut suivre une méthode personnelle ou scientifique, elle fait l'objet de critiques, d'analyses et d'évaluations constantes et, plus que tout, elle maintient un lien permanent et étroit avec la réalité dont elle constitue le miroir - Stendhal l'a ainsi exprimé plus ou moins déformant. Lieu par excellence aussi de la quête, qu'elle soit éthique ou métaphysique - Les Pensées de Montaigne nous en fournissent un bel exemple - ou qu'elle se confonde avec la recherche - même des formes de questionnement de son propre objet, comme chez Proust - la littérature a une troisième grande caractéristique, une troisième grande qualité : celle de pouvoir déclencher un processus d'imagination qui n'est pas prédéterminé, et qui procède toujours d'un mouvement humain de multiples intercessions : le temps, l'histoire et l'historicité, la mémoire.

Ce mouvement est transversal à plusieurs domaines de connaissance et toujours prêt à renaître de ses cendres. Ce n'est pas n'importe quel phénix. C'est le 'beau phénix' chanté par Apollinaire et ressurgi sous la plume de Doris Lessing pour nous rappeler que le poète est voleur - alliant les deux significations du mot - de feu, prêt à céder le flambeau à ceux qui voudront bien participer au processus de construction du sens - d'autant plus in-fini et en devenir qu'il est humain. For good and for ill nous rappelle l'auteure anglaise nobélisée... avec la noblesse du bel oiseau de feu. 


\section{Bibliographie}

BACHELARD, Gaston (1971). La poétique de la rêverie, Paris : PUF.

DAMÁsıo, António (1995). L'erreur de Descartes. La raison des émotions, Paris : Odile Jacob.

DAMASIO, António (1999). Le sentiment même de soi. Corps, émotions, conscience, Paris : Odile Jacob.

Eco, Umberto (1970). "Le problème de la réception ". In : Critique sociologique et critique psychanalytique, Ed. de l'Institut de Sociologie, Université Libre de Bruxelles.

GenetTE, Gérard (1972). Figures III, Paris : Seuil.

LESSING, Doris (2007). "Nobel lecture". In : http://nobelprize.org/nobel_prizes/literature/laureates/2007/lessing-lecture.html consulté le 04/01/2008].

LESSING, Doris (1994). L'œuvre de l'art. Immanence et transcendance. Paris: Seuil.

LYOTARD, Jean-François (1979). La condition postmoderne, Paris : Minuit.

LYOTARD, Jean-François (1988). Le postmoderne expliqué aux enfants, Paris : Galilée.

MALLARME, Stéphane (1998). CEuvres complètes, édition présentée, établie et annotée par Bertrand Marchal, Paris : Gallimard, coll. Bibliothèque de la Pléiade, tome I.

MALLARME, Stéphane (2003). CEuvres complètes, édition présentée, établie et annotée par Bertrand Marchal, Paris : Gallimard, coll. Bibliothèque de la Pléiade, tome II.

MALLARME, Stéphane (1995). Correspondance, Lettres sur la poésie éd. Bertrand Marchal (Préface d'Yves Bonnefoy), Paris : Gallimard, coll "Folio-Classique".

SALMON, Christian (2007). Storytelling. La machine à fabriquer des histoires et à formater les esprits, Paris : Éd. La Découverte.

SAlmon, Christian (2005). Verbicide, Du bon usage des cerveaux humains disponibles, Castelnau le Lez : Ed. Climats.

SHERMER, Michael, (2007). "The Really Hard Science”. In: Scientific American, October 2007 Issue [disponible à l'adresse http://www.scientificamerican.com/article.cfm?id=the-really-hard-science].

VAleRY, Paul (1957). "Magie", “Mélanges". In : CEuvres, I, édition établie et annotée par Jean Hytier, Paris : Gallimard, coll. Bibliothèque de la Pléiade. 